\title{
Lymphadenectomy in the Treatment of Colon Cancer: A Survival Analysis
}

\author{
Romualdo Silva Corrêa ${ }^{1}$, Luciana Ayres De Oliveira Limaa ${ }^{2}$, Isa Maryana Araújo Bezerra De Macedo², Amália \\ Cinhtia Meneses Rêgo ${ }^{3}$ and Irami Araújo Filho ${ }^{4 *}$
}

${ }^{1}$ Coloproctologist Surgeon, Brazil

${ }^{2}$ Undergraduate Student of Medicine at Potiguar University, Brazil

${ }^{3}$ Postgraduate Program in Biotechnology at Potiguar University, Brazil

${ }^{4}$ Postgraduate Program in Biotechnology at Potiguar University, Brazil

*Corresponding author: Irami Araújo Filho, Postgraduate Program in Biotechnology at Potiguar University, Brazil.
Received Date: January 08, 2020

Published Date: January 14, 2020

\begin{abstract}
Colon cancer is a curable disease when restricted to the bowel and colectomy, the primary treatment. However, the presence and number of resected lymph nodes influence the therapeutic approach and prognosis of the patient. To evaluate the impact of the number of resected lymph nodes on the overall survival of patients treated for colon cancer at the League of Cancer Hospital - Natal - State of Rio Grande do Norte (RN) - Northeast Brazil. A retrospective observational study of 80 patients with colon cancer from Dr. Luiz Antônio Hospital (Natal-RN / Brazil), considering the period 2007-2014. Data were collected through medical records review. Survival rates were calculated and compared using the non-parametric Kaplan-Meier and Wilcoxon tests, respectively. All patients underwent radical surgical treatment associated or not with chemotherapy and/or radiotherapy treatment. The median survival time for the group of patients who had 12 or more resected lymph nodes was 9.4 years, in contrast to the 3.3 years of those who had less than 12 lymph nodes. Conclusion: It was concluded that a total of 12 or more resected lymph nodes confirmed by histopathology is associated with increased long-term survival in patients with colon cancer undergoing radical colectomy with or without chemotherapy and radiotherapy.
\end{abstract}

Keywords: Colonic neoplasms; Colorectal surgery; Lymph nodes; Lymphadenectomy; Lymph node excision; Survival analysis

\section{Introduction}

Colon cancer (CC) is one of the most prevalent neoplasms in the world. In Brazil, it is the fourth most common cancer. In 2018 the estimate of new cases was 36.360 (17.380 men/18.980women) [1]. Notwithstanding the high mortality, it is a curable disease when localized and restricted to the intestine [2]. The best therapeutic results are achieved through standard treatment, colectomy associated with lymphadenectomy (radical surgery). Cure occurs in up to $50 \%$ of patients when considering all stages of the disease. Nevertheless, relapse is still a major problem, contributing to the increase in deaths [3-5]. The prognosis and survival of each patient depend on correct staging, with TNM being the most used system. In this, the classification of " $\mathrm{N}$ " is given by the absolute number of metastatic lymph nodes (LN), where $\mathrm{N} 1$ will be considered from one to three lymph nodes and N2 above this value [6]. The presence of this lymph vascular invasion can distinguish stages
II and III and alter the proposed treatment. According to current clinical evidence, adjuvant chemotherapy in the treatment of stage II-CC does not show an increase in survival, whereas, in stage III, its performance is indicated [4]. From this perspective, there would be impaired patient care if there were errors in lymph node count or histopathological positivity for malignancy [7].

Assessment of lymph node status is an alternative method, and one of the independent factors for the prognosis of WC. This is obtained by histopathological examination after radical surgery, in which reports express the number of resected lymph nodes (LNR) and the number of lymph nodes positive for tumor presence (LN+) [1,7-9]. Thus, in addition to lymphatic and vascular invasion, metastasis-free lymph nodes are considered a good prognostic factor. In addition to establishing proportionality, lymph node histological analysis provides additional information, such as the 
presence of extranidal extension (perinodal tumor cells), directly related to tumor aggressiveness [10].

The American Joint Committee on Cancer (AJCC) and the National Cancer Institute recommend that a minimum of 12 lymph nodes be examined in patients with $\mathrm{CC}$ to confirm the absence of lymph node involvement by the tumor [6]. This recommendation considers the number of LNs analyzed to be reflex of the radicality of lymph vascular mesenteric dissection in surgical resection and histopathological in the surgical specimen. In addition to being decisive for the elaboration of the therapeutic strategy of CC, the values of LNR and LN + are independent predictors that interfere with cancer outcomes. The overall survival and the likelihood of relapse, thus contributing to the definition of prognosis [11-13].

In this sense, it is essential to define the minimum number of LNs to be examined in a surgical specimen to determine the patient's lymph node status as accurately as possible and to avoid sub staging, as the therapeutic approach is based on staging tumor completely. Based on the above, the present study evaluated the impact. It discussed the importance of quantifying the number of resected lymph nodes in the overall survival of patients diagnosed and treated for Colon Cancer in Natal, League of Cancer Hospital State of Rio Grande do Norte. (RN) - Northeast Brazil.

\section{Methods}

Aretrospective observational study of 80 patients was carried out at Dr. Luiz Antônio Hospital / League Against Cancer, a referral cancer hospital located in Natal - State of Rio Grande do Norte / Northeast Brazil, referring to the period between the years. From 2007-2014. The study was approved by the Research Ethics Committee of the League Against Cancer under protocol 044/044/2009, registered at Plataforma Brazil/National Commission of Research Ethics CONEP/Ministry of Health. The study included all patients aged over 18 years with colon adenocarcinoma, confirmed by previous histopathological examination, who underwent radical surgery as a curative treatment, associated or not with radiotherapy and/or chemotherapy. Data were collected by reviewing medical records considering the following information: age, gender, origin, diagnosis, date of diagnosis (time of biopsy), TNM staging, type of surgical treatment, adjuvant treatment, disease recurrence, death's age, and cause of death. All patients underwent surgical treatment alone or associated with chemotherapy and/or radiotherapy performed by members of the medical staff of Dr. Luiz Antônio Hospital. Cancer staging was performed following the TNM system, according to the American Joint Committee on Cancer. Overall survival was calculated considering the date of diagnosis. The non-parametric Kaplan-Meier and Wilcoxon methods were used to estimate and compare the overall survival rate using SPSS/IBM® version 25 statistical data analysis software. The results of the Wilcoxon test evidence were evaluated, considering the significance statistics level 5\%.

\section{Results}

The sample of 80 patients was divided into three age groups: $15 \%$ aged $<50$ years, $47.5 \%$ aged $50-70$ years, and $37.5 \%$ aged $>70$ years. Regarding the epidemiological characteristics, the analysis of the sample group revealed that most of the patients were women from the interior of the state of NB. Regarding the clinical features, it was noticed that the incidence of CC was higher in the right colon. Regarding lymphadenectomy (radical colectomy), 70\% of the patients had 12 or more LN analyzed in the histopathological examination of the surgical specimen, $15 \%<12$ LNR, and in the remaining $15 \%$ there was no detection of $L N$ by the pathologist (Table 1).

Table 1: Epidemiological characteristics of patients with colon cancer.

\begin{tabular}{|c|c|c|}
\hline $\begin{array}{l}\text { Epidemiological } \\
\text { Characteristics }\end{array}$ & Number of Patients & Percentage (\%) \\
\hline \multicolumn{3}{|c|}{ Age Group } \\
\hline$<50$ & 12 & 15 \\
\hline $50-70$ & 38 & 47.5 \\
\hline$>70$ & 30 & 37.5 \\
\hline \multicolumn{3}{|c|}{ Sex } \\
\hline Male & 30 & 37.5 \\
\hline Female & 50 & 62.5 \\
\hline \multicolumn{3}{|c|}{ Provenance } \\
\hline Main City/Capital & 22 & 27.5 \\
\hline Inland cities & 58 & 72.5 \\
\hline \multicolumn{3}{|l|}{ Colon Segment } \\
\hline Right & 58 & 72.5 \\
\hline Left & 22 & 27.5 \\
\hline \multicolumn{3}{|c|}{ Resected Lymph Nodes } \\
\hline$<12$ & 12 & 15 \\
\hline$\geq 12$ & 56 & 70 \\
\hline $\begin{array}{l}\text { No lymph nodes } \\
\text { resected }\end{array}$ & 12 & 15 \\
\hline
\end{tabular}

Regarding treatment, besides curative surgery, $62.4 \%$ of patients underwent adjuvant therapy, 3.8\% neoadjuvant treatment, $3.8 \%$ concomitant therapy, and $30 \%$ no (Table 2 ).

Table 2: Distribution of patients with colon cancer according to therapy associated with surgical treatment and survival.

\begin{tabular}{|c|c|c|}
\hline Treatment & Number of Patients & Percentage (\%) \\
\hline None & 24 & 30 \\
\hline Neoadjuvant & 3 & 3.8 \\
\hline Adjuvant & 50 & 62.4 \\
\hline Concomitant & 3 & 3.8 \\
\hline \multicolumn{2}{|c|}{ Neoadjuvant Treatment } \\
\hline QT & 2 & 33.3 \\
\hline RxT & 2 & 33.3 \\
\hline QT+ RxT & 2 & 33.3 \\
\hline Adjuvant Treatment & 22 & 27.5 \\
\hline QT & 47 & 88.7 \\
\hline RxT & 1 & 1.9 \\
\hline QT+ RxT & 5 & 9.4 \\
\hline Death & & 65 \\
\hline Yes & 52 & 35 \\
\hline Not & 28 & \\
\hline
\end{tabular}


There was a statistically significant difference between the analyzed groups ( $p$-value $=0.005$ ) so that patients who had 12 or more resected lymph nodes had more prolonged survival compared to the other groups (Table 3).

Table 3: Patient survival according to the number of lymph nodes resected.

\begin{tabular}{|c|c|c|c|}
\hline $\begin{array}{c}\text { Resected } \\
\text { Lymph Nodes }\end{array}$ & $\begin{array}{c}\text { Survival } \\
\text { (average/years) }\end{array}$ & $\begin{array}{c}\text { Confidence } \\
\text { interval (95\%) }\end{array}$ & (p-value) \\
\hline$\geq 12$ & 9.4 & $7.9-10.9$ & 0.005 \\
\hline$<12$ & 3.3 & $1.1-5.5$ & \\
\hline None & 3.8 & $1.9-5.6$ & \\
\hline
\end{tabular}

The median survival time was 9.4 years for this group of patients in contrast to 3.3 years for patients who had less than 12 resected lymph nodes (Figure 1).

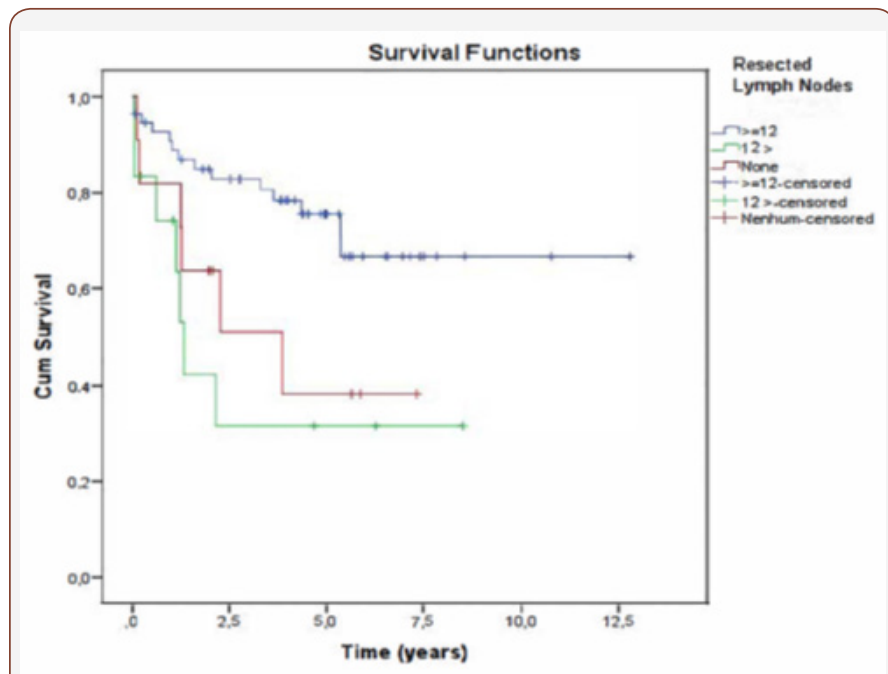

Figure 1: Estimated overall survival curve concerning the number of resected lymph nodes.

\section{Discussion}

The importance of the evaluation of resected lymph nodes in predicting the prognosis of patients with colorectal cancer is increasingly consolidated. The discussion about the objective of the study is how many lymph nodes would be necessary to dry during the surgery and to recover in the surgical specimen during the histopathological examination to obtain reliable results [14]. The main factors that may interfere in the evaluation of the number of NRLs are the surgical technique. methods applied in the pathology and characteristics of the patient (age; stage of the disease; among others) [15-17]. Regarding the surgical technique, the best results are attributed to complete mesocolic excision. The increased extension of the obtained surgical specimen promotes the resection of a more significant number of lymph nodes and provides sufficient material for one for adequate histological analysis. This fact does not increase perioperative morbidity and is associated with improved overall survival in stages I-III [18].

In the present study, all patients were operated by the same team of surgeons. who underwent standard surgical technique and opted for a broad and radical approach, reserving complete mesocolic excision in cases of rectal carcinoma. Histopathological analyses were similar in all cases, considering that the anatomical specimens were examined by the same group of pathologists who use standardized techniques and follow strict institutional protocols. Thus, possible biases that could exist in multicenter studies may be disregarded in the results presented [19]. Regarding the clinical characteristics of the patients, the distribution by age and sex is in agreement with the literature. as well as the survival rates. It is noteworthy that the present study considered the excision pattern of at least 12 lymph nodes for all age groups. as provided by the AJCC. However, some articles in the literature already consider age as an essential factor in determining the number of lymph nodes $[20,21]$.

A Chinese study concluded that more than nine lymph nodes would be enough for patients over 80 years old, finding similar results concerning prognosis and survival [16]. The possible explanation would be the fact that the elderly have a modulated immune and inflammatory response concerning cancer and, thus, there is less involvement of regional lymph nodes by tumor cells. Moreover, in elderly patients, there is lymph node involution with aging [16].

On the other hand, a study involving 2360 patients younger than 40 years showed that resection of more than 22 lymph nodes was able to increase survival by $4 \%$ [17]. Although not the most prevalent age group for colorectal câncer, the diagnosis occurred at an advanced stage, with a higher number of affected lymph nodes, aggressive histological subtypes, which required more extensive intestinal resections and a more significant number of ganglia.

As for the stage of the disease, it is crucial to consider that the more advanced câncer, the more LN will be affected by the tumor, becoming larger and facilitating its resection. The explanation lies in the fact that the more massive disease, the deeper the intestinal wall penetration and mesenteric invasion, leading to a higher antigenic immune and inflammatory response in the regional lymph nodes. This promotes an increase in lymph node number and volume, but not necessarily metastatic positivity. Thus, carcinomas with T3 and T4 staging tend to have a higher number of resected lymph nodes [18].

A survey by Chandrasinghe et al. collected data from 131 patients with stage II and III CC who underwent curative resection surgery and whose surgical specimens had at least five lymph nodes evaluated in the histopathological examination. Prospectively analyzing the cancer outcomes, it was concluded that in patients whose surgical specimen had 14 or more NRLs, there was a better overall survival benefit ( $p=0.005$ ) compared to the other groups; rectal $(n=83 / p=0.03)$ and colorectal $(n=46 / p=0.08)$ cancer. Also, the number of LNs analyzed has been shown to affect postoperative survival in patients with or without $\mathrm{LN}+$, concerning the more resected NLR, the better the long-term prognosis [20].

Downing et al. collected information from 128.071 patients diagnosed with colon adenocarcinoma from The Surveillance. 
Epidemiology. and End Results database from 1988-2005. The authors concluded that the optimal minimum number of resected NRLs for patients with and without $\mathrm{LN}+$, a significant predictor of survival, is between 10-15 LN. It was also observed that the survival rate benefit in all analyzed subgroups continues to increase in proportion to the higher number of resected NRNs. indicating that patients continue to benefit at the rate that more NRs are examined. even after the optimal value established [21].

Rivadulla-Serrano et al. analyzing data from 148 patients diagnosed with CC / N0 and undergoing curative surgery between 1995-2001, concluded that dissection of a larger number of lymph nodes is related to improved survival rates in 05 years [11]. This conclusion was inferred since the rate in question was $63 \%$ in the group with $<7$ NLR. $80.6 \%$ in patients with 7-14 NLR and $91.8 \%$ in those $>14$ NLR ( $p<0.01$ ). Thus, the study recommended to obtain the largest amount of NLR possible and not to establish a minimum number of NL to be resected in operation and analyzed by histopathological examination [22].

Finally, Chen et al. concluded that the number of LN analyzed by histopathology during the examination of the surgical specimen correlates with clinical improvement and survival in patients with CC-N0 [23]. The study sample comprised data stored in the Surveillance, Epidemiology, and end Results of 82.896 patients who underwent CC curative surgery between the years 1988-2000. In this study, the sample was divided into four subgroups of patients based on the number of LN examined postoperatively: $0 ; 1-7 ; 8-15$ and $>15$ LNR. For all tumor stages, the increase in the number of lymph nodes evaluated in the histopathological examination increased the survival rate. Patients with less than $15 \mathrm{LN}$ analyzed compared to the subgroup that had 1-7 LN resected experienced a $20.6 \%$ reduction in mortality regardless of other patient and tumor stage variables [21-23].

The results achieved by the studies described above validate the findings observed in the present research. Postoperative survival rates increased exponentially and proportionally to the increase in the number of LNR, confirming the fact that the LNR value is relevant for both correct CC staging and prognosis and survival prediction.

\section{Conclusion}

In conclusion, the accurate staging and determination of a correct therapeutic strategy for colorectal cancer patients justify the need for adequate sampling and histopathological examination of a sufficient number of LNs. This study confirmed that a total of 12 or more resected LNs is associated with increased longterm survival. In this sense, it is essential to perform the broadest possible resection, which is possible in most cases of colon cancer by standardized and scientifically validated methods.

\section{Acknowledgment}

The authors thank the Chief Surgeon and Full Professor, Department of Surgery, Federal University of Rio Grande Norte/ Brazil, Prof. Dr. Aldo da Cunha Medeiros, for his contribution and relevance scientific discussion and the supervision of this research, acting as an expert consultant on the bibliographic survey, analysis, and scientific advice. We also thank all the study components for their dedication and effort to build a scientifically validated quality study.

\section{Conflict of Interest}

There are no conflicts of interest to declare by any of the authors of this study.

\section{References}

1. Parsons HM, Tuttle TM, Kuntz KM, Begun JW, Mc Govern PM, et al. (2011) Association between lymph node evaluation for colon cancer and node positivity over the past 20 years. JAMA 306(10): 1089-1097.

2. Ferlay J, Soerjomataram I, Ervik M, Dikshit R, Eser S, et al. (2015) Cancer incidence and mortality worldwide: sources, methods and major patterns in GLOBOCAN 2012. Int J Cancer 136(5): E359-E386.

3. Chang GJ, Rodriguez Bigas MA, Skibber JM, Moyer VA (2007) Lymph node evaluation and survival after curative resection of colon cancer: systematic review J Natl Cancer Inst 21 99(6): 433-441.

4. Paquette IM, Madoff RD, Sigurdson ER, Chang GJ (2018) Impact of Proximal Vascular Ligation on Survival of Patients with Colon Cancer. Ann Surg Oncol 25(1): 38-45.

5. Wright FC, Law CH, Berry S, Smith AJ (2009) Clinically important aspects of lymph node assessment in colon cancer. J Surg Oncol 99(4): 248-255.

6. Garcia B, Guzman C, Johnson C, Hellenthal NJ, Monie D, Monzon JR (2016) Trends in lymph node excision and impact of positive lymph node ratio in patients with colectomy for primary colon adenocarcinoma: Population based study 1988 to 2011. Surg Oncol 25(3): 158-163.

7. Märkl B (2015) Stage migration vs immunology: The lymph node count story in colon cancer. World J Gastroenterol 21(43): 12218-12233.

8. Amri R, Klos CL, Bordeianou L, Berger DL (2016) The prognostic value of lymph node ratio in colon cancer is independent of resection length. Am J Surg 212(2): 251-257.

9. Le Voyer TE, Sigurdson ER, Hanlon AL, Mayer RJ, Macdonald JS, et al. (2003) Colon cancer survival is associated with increasing number of lymph nodes analyzed: a secondary survey of intergroup trial INT-0089. J Clin Oncol 21(15): 2912-2919.

10. Patt DJ, Brynes RK, Vardiman JW, Coppleson LW (1975) Mesocolic lymph node histology is an important prognostic indicator for patients with carcinoma of the sigmoid colon: an immunomorphologic study. Cancer 35(5): 1388-1396.

11. Hashiguchi Y, Hase K, Ueno H, Mochizuki H, Kajiwara Y, et al. (2010) Prognostic significance of the number of lymph nodes examined in colon câncer surgery: clinical application beyond simple measurement. Ann Surg 251(5): 872-881.

12. Compton CC, Fielding LP, Burgart LJ, Conley B, Cooper HS, et al. (2000) Prognostic factors in colorectal cancer. College of American Pathologists Consensus Statement 1999. Arch Pathol Lab Med 124(7): 979-994.

13. Bertelsen CA (2017) Complete mesocolic excision an assessment of feasibility and outcome. Dan Med J 64(2).

14. Guan X, Chen W, Jiang Z, Liu Z, Miao D, et al. (2016) Exploration of the Optimal Minimum Lymph Node Count after Colon Cancer Resection for Patients Aged 80 Years and Older. Sci Rep 6: 38901.

15. Guan X, Wang Y, Hu H, Zhao Z, Jiang Z, et al. (2018) Reconsideration of the optimal minimum lymph node count for young colon câncer patients: a population-based study. BMC Cancer 18(1): 623.

16. Tsai HL, Huang CW, Yeh YS, Ma CJ, Chen CW, et al. (2016) Factors affecting number of lymph nodes harvested and the impact of examining a minimum of 12 lymph nodes in stage I-III colorectal cancer patients: a retrospective single institution cohort study of 1167 consecutive patients. BMC Surg 16: 17. 
17. Wright FC, Law CH, Last L, Khalifa M, Arnaout A, et al. (2003) Lymph node retrieval and assessment in stage II colorectal cancer: apopulationbased study. Ann Surg Oncol 10(8): 903-909.

18. Angelis A, Montibeller G, Hochhauser D, Kanavos P (2017) Multiple criteria decision analysis in the context of health technology assessment: a simulation exercise on metastatic colorectal cancer with multiple stakeholders in the English setting. BMC Med Inform Decis Mak 17(1): 149.

19. Brenner H, Gefeller O, Hakulinen T (2004) Period analysis for up to date câncer survival data: theory, empirical evaluation, computational realisation and applications. Eur J Cancer 40(3): 326-335.

20. Chandrasinghe PC, Ediriweera DS, Hewavisenthi J, Kumarage S, Deen KI (2014) Total number of lymph nodes harvested is associated with better survival in stages II and III colorectal cancer. Indian J Gastroenterol 33(3): 249-253.

21. Downing SR, Cadogan KA, Ortega G, Jaji Z, Bolorunduro OB, et al. (2010) The number of lymph nodes examined debate in colon cancer: how much is enough? J Surg Res 163(2): 264-269.

22. Rivadulla Serrano MI, Martínez Ramos D, Armengol Carrasco M, Escrig Sos J, Paiva Coronel GA, et al. (2010) Impact of the total number of harvested lymph nodes after colon cancer resections on survival in patients without involved lymph node. Rev Esp Enferm Dig 102(5): 296301.

23. Chen SL, Bilchik AJ (2006) More extensive nodal dissection improves survival for stages I to III of colon cancer: a population-based study. Ann Surg 244(4): 602-610. 